\title{
Effect of LTI on the microstructure and mechanical properties of biodegradable PLLA/PBSC blend
}

\author{
Vilay Vannaladsaysy ${ }^{1,2}$, Mitsugu Todo $^{1}$, Mariatti Jaafar ${ }^{2}$ \\ ${ }^{I}$ Research Institute for Applied Mechanics, Kyushu University, Kasuga, Fukuoka, Japan \\ ${ }^{2}$ School of Materials \& Mineral Resources Engineering, Universiti Sains Malaysia, 14300 Nibong Tebal, \\ Pulau Pinang, Malaysia
}

\begin{abstract}
Blends of poly (L-lactic acid) (PLLA) and poly (butylene succinate-co-e-caprolactone) (PBSC) were prepared and 2 wt.\% of lysine triisocyanate (LTI) was selected as a compatibilizer for each blends. The materials were characterized by FTIR and FE-SEM and mode I fracture energy was evaluated. It was found that the LTI addition effectively improves the mode I fracture energy of the blends. It was supported by morphological analyses that the brittle deformation behavior of neat PLLA changes to ductile deformation with presence of elongated fibril structures in the blend systems.

Keywords: Polymer blend, Biodegradability, Mechanical property, Blend morphology

\section{Introduction}

Recently, much attention has been paid to PLLA, because of its biodegradability and bioabsorbability [1]. However, PLLA exhibits brittle fracture behavior in nature and therefore, the toughening of PLLA becomes one of the most important issues in the field of bio-polymer engineering [2]. Several approaches have been used to improve the mechanical properties of PLLA, including polymer blending with a ductile polymer that is known as an effective way to improve the toughness of a base brittle polymer [2-4].

In this study, PLLA/PBSC polymer blends were developed with 2 wt.\% of LTI. The purpose of this study is to assess the effects of LTI addition on the immiscibility between the polymer components and on the fracture property, and also characterize the relationship between the microstructural modification and the fracture property.
\end{abstract}

\section{Experimental}

\subsection{Materials and specimen}

PLLA pellets (Lacty@ $\# 5000, \mathrm{M}_{\mathrm{w}}=1.45 \times 10^{5}$, Toyota Motor Co., Ltd) and PBSC pellets $\left(\mathrm{M}_{\mathrm{w}}=1.7 \times 10^{5}\right.$, Daicel Chemical Industries, Ltd.) were blended using a conventional melt-mixer at $190^{\circ} \mathrm{C}$ and a rotor speed of 50 $\mathrm{rpm}$ for $20 \mathrm{~min}$. The blend ratios of PBSC were chosen to be 10,20 and 30 wt.\%. High viscous solution of LTI (Kyowa Co., Ltd.) was also added during blending process. The mixtures were then molded at $30 \mathrm{MPa}$ and $190^{\circ} \mathrm{C}$ using a hot press, and then followed by cooling process to produce the sheets of $140 \times 140 \times 2 \mathrm{~mm}^{3}$. The single edge notched bend (SENB) specimens were then prepared from the sheets. PLLA/PBSC and PLLA/PBSC/LTI with 10wt.\% PBSC are denoted thereafter as $\mathrm{AC}_{10}$ and $\mathrm{ACI}_{10}$, respectively.

\subsection{Measurements}

The Fourier Transform Infra-red (FT-IR) measurement was carried out using Bio-Rad FTS-6000 spectrometer with resolution of $8 \mathrm{~cm}^{-1}$ for 32 scans over wave number range of $400-4000 \mathrm{~cm}^{-1}$. Film samples were obtained by casting polymer resolution on the $\mathrm{KBr}$ disc.
The mode I fracture tests of the SENB specimens were performed at $1 \mathrm{~mm} / \mathrm{min}$ of loading-rate using a servohydraulic testing machine. The histories of load and displacement at the loading-point were recorded using a digital recorder and the load-displacement relations were obtained. The average mode I fracture energy, $J_{f}$, was then evaluated by using the following formula:

$$
J_{f}=\frac{U_{f}}{B(W-a)}
$$

where $U_{\mathrm{f}}$ is the total fracture energy that is dissipated by the complete fracture of the specimen. Thus, $J_{f}$ is recognized as the average energy per unit area of fracture surface.

The morphology of the blends was investigated by observing cryo-fractured surfaces of the specimens using a high-resolution field emission scanning electron microscope (FE-SEM) to characterize the microstructural modification. The fractured surfaces of the SENB specimens were also observed by FE-SEM to characterize the fracture micromechanism and the effect of LTI addition on the fracture behavior.

\section{Results and discussion \\ 3.1. Microstructure}

As typical examples, the cryo-fractured surfaces of $\mathrm{AC}_{30}$ and $\mathrm{ACI}_{30}$ are shown in Fig.1. A phase separation characterized by dispersing large spherical structures in the matrix is clearly observed on $\mathrm{AC}_{30}$, and the spherical structures are thought to be the spherulites of PBSC. On the contrary, $\mathrm{ACl}_{30}$ does not exhibit such spherulites, indicating that the immiscibility between PLLA and PBSC was dramatically improved by LTI addition.

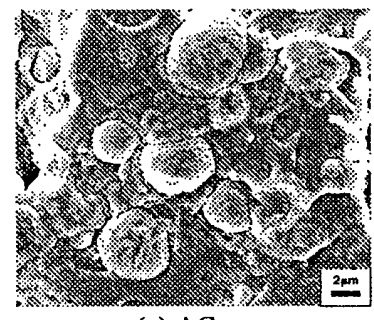

(a) $\mathrm{AC}_{30}$

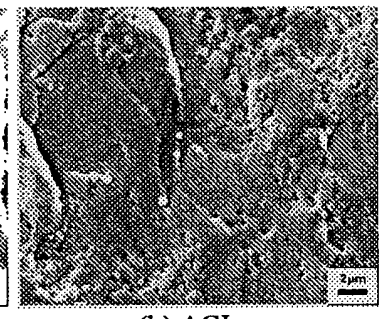

(b) $\mathrm{ACI}_{30}$
Fig.1 FE-SEM micrographs of cryo-fractured surfaces.

\subsection{FT-IR analysis}

Fig. 2 shows the FT-IR spectra of PLLA, PBSC, LTI, $\mathrm{AC}_{20}$ and $\mathrm{ACI}_{20}$. It is seen that PLLA, PBSC and the blends exhibited very similar spectra patterns with the $\mathrm{C}=\mathrm{O}$ peak at about $1700 \mathrm{~cm}^{-1}$. However, the $\mathrm{C}=\mathrm{O}$ peak of PBSC (d) was much higher than that of PLLA (a). It is therefore understood that the higher $\mathrm{C}=\mathrm{O}$ peak of $\mathrm{AC}_{20}$ (b) than PLLA was a result of PBSC blending. It is clearly seen that the $\mathrm{C}=\mathrm{O}$ peak of $\mathrm{ACI}_{20}$ (c) became lower than that of $\mathrm{AC}_{20}$, suggesting that the mobilities of PLLA and PBSC 
molecules were reduced due to LTI addition. On the other hand, the spectra of LTI (e) was obviously characterized by the existence of the large NCO peak around $2200 \mathrm{~cm}^{-1}$. It is noted that there was no apparent peak of NCO in the spectra of $\mathrm{ACI}_{20}$, indicating that $\mathrm{NCO}$ groups were chemically reacted with $\mathrm{OH}$ groups existing at the ends of PLLA and PBSC molecules. This kind of chemical reaction between $\mathrm{NCO}$ and $\mathrm{OH}$ groups is called urethane bonding [ ]. It is thus concluded from these FT-IR results that LTI works as a compatibilizer connecting between PLLA and PBSC molecules.

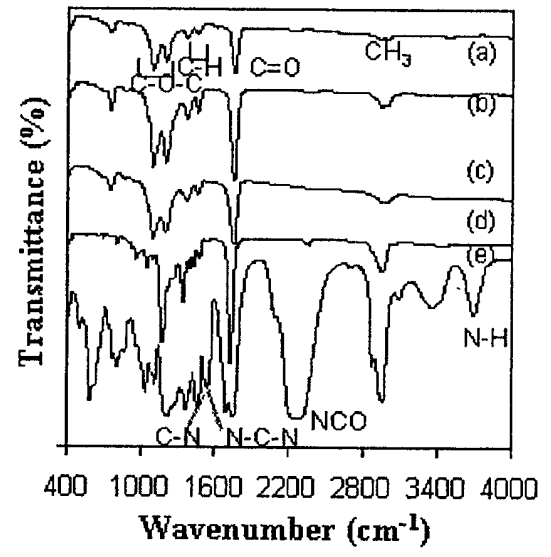

Fig. 2. FT-IR spectra of (a) PLLA, (b) $\mathrm{AC}_{20}$, (c) $\mathrm{ACI}_{20}$, (d) PBSC and (e) LTI.

\subsection{Mode I fracture energy}

Averaged total fracture energy per unit fracture surface area, $J_{f}$, is shown in Fig.3. The $J_{f}$ values of PLLA/PBSC blends are higher than that of neat PLLA, indicating the effectiveness of blending. The graph suggests that $J_{f}$ of PLLA/PBSC reached is maximized with $20 \mathrm{wt} . \%$ of PBSC. On the other hand, $J_{f}$ of PLLA/PBSC/LTI almost linearly increased with increase of PBSC content. It is noted that the highest $J_{f}$ value of PLLA/PBSC/LTI in this experiment was approximately $40 \mathrm{~kJ} / \mathrm{m}^{2}$ that was about twice of the maximum $J_{f}$ of PLLA/PBSC, that is, $20 \mathrm{~kJ} / \mathrm{m}^{2}$.

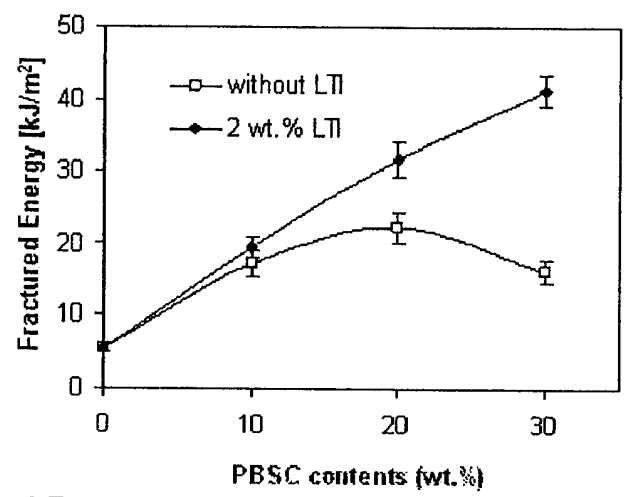

Fig. 3 Fracture energy as a function of PBSC content.

\subsection{Fracture surface morphology}

FE-SEM micrographs of fracture surfaces in the crack propagation regions are shown in Fig.4. It is noted that these fracture surfaces of PLLA/PBSC blends are much rougher than that of neat PLLA that exhibits very smooth surface, corresponding to the higher energy dissipation on the fracture surfaces of the blends than that of PLLA. It is apparent that the elongation of the fibril structures in $\mathrm{AC}$ blends seems to be larger than that in ACI blends. Many voids are also observed on the $\mathrm{AC}$ surfaces; on the other hand, such voids do not exist on the ACI surfaces. Thus, this kind of voids are thought to be formed as the traces of removed PBSC spherulites. Such voids cause localized stress concentrations in the surrounding regions, resulting in acceleration of fracture initiation. Therefore, the fracture initiation in ACI blends without any voids is much slower than that in AC blends. It is concluded that this kind of delay of fracture initiation results in the improved fracture energy as shown in Fig.3.

\section{Conclusions}

PLLA/PBSC and PLLA/PBSC/LTI polymer blends were developed and examined in order to investigate the effects of LTI addition on the microstructure and the facture property. FT-IR and FE-SEM analyses and mode I fracture testing were performed to characterize the relationship between the micro- and macroscopic properties. It was found that LIT addition dramatically improves the immiscibility due to chemical reaction between NCO and $\mathrm{OH}$ groups, resulting in the effective improvement of the mode I fracture energy.

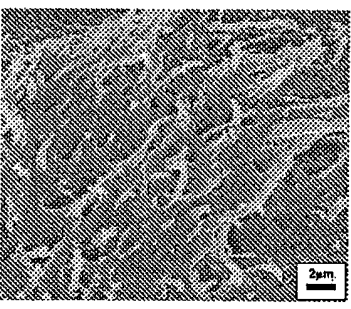

(a) $\mathrm{AC}_{10}$

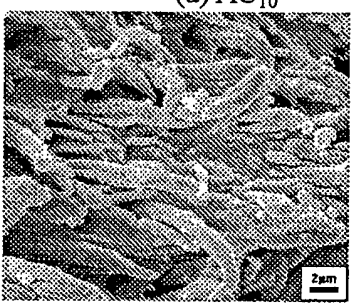

(c) $\mathrm{AC}_{20}$

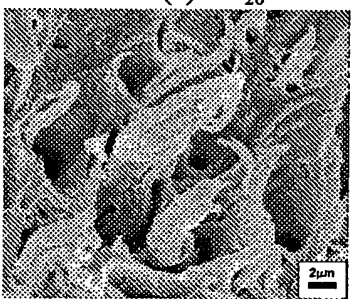

(e) $\mathrm{AC}_{30}$

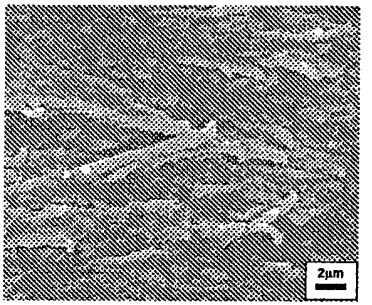

(b) $\mathrm{ACI}_{10}$

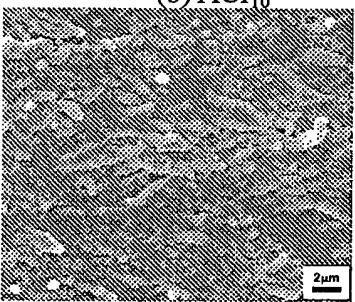

(d) $\mathrm{ACI}_{20}$

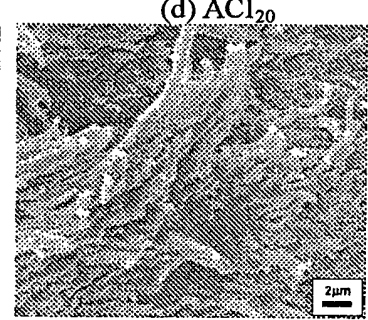

(f) $\mathrm{ACI}_{30}$
Fig. 4 FE-SEM micrographs of mode I fracture surfaces.

\section{Acknowledgements}

This work was funded by AUN/SEED-Net and JCA is gratefully acknowledged. The authors greatly acknowledge the support from Kyushu University and Universiti Sains Malaysia.

\section{References}

[1] M. Todo, et al., J Mater Sci Lett 21 (2202) 1203.

[2] M. Todo, et al., Eng Frac Mech 74(2007) 1872.

[3] M. Shibata, et al., Polymer. 47 (2006) 3557.

[4] M. Shibata, et al., Polymer. 48 (2007) 2768.

[5] Scott A. Guelcher, et al., Biomaterials 29 (2008) 1762. 